FERMILAB-Conf-94/100

\title{
Fluorescent Compounds for Plastic Scintillation Applications
}

\author{
Anna Pla-Dalmau and Alan D. Bross \\ Fermi National Accelerator Laboratory \\ P.O. Box 500, Batavia, Illinois 60510
}

April 1994

Presented at the MRS Symposium on Scintillator and Phosphor Materials, San Francisco, California, April 4-8, 1994 


\section{Disclaimer}

This report was prepared as an account of work sponsored by an agency of the United States Government. Neither the United States Government nor any agency thereof, nor any of their employees, makes any warranty, express or implied, or assumes any legal liability or responsibility for the accuracy, completeness, or usefulness of any information, apparatus, product, or process disclosed, or represents that its use would not infringe privately owned rights. Reference herein to any specific commercial product, process, or service by trade name, trademark, manufacturer, or otherwise, does not necessarily constitute or imply its endorsement, recommendation, or favoring by the United States Government or any agency thereof. The views and opinions of authors expressed herein do not necessarily state or reflect those of the United States Government or any agency thereof. 


\title{
FLUORESCENT COMPOUNDS FOR PLASTIC SCINTILLATION APPLICATIONS
}

\author{
ANNA PLA-DALMAU AND ALAN D. BROSS \\ Fermi National Accelerator Laboratory, P.O. Box 500, Batavia, IL 60510
}

\begin{abstract}
Several 2-(2'-hydroxyphenyl)benzothiazole, -benzoxazole, and -benzimidazole derivatives have been prepared. Transmittance, fluorescence, light yield, and decay time characteristics of these compounds have been studied in a polystyrene matrix and evaluated for use in plastic scintillation detectors. Radiation damage studies utilizing a ${ }^{60}$ Co source have also been performed.
\end{abstract}

\section{INTRODUCTION}

New applications for plastic scintillation detectors have resulted from recent developments in both scintillating plastic optical fibers and photon detection devices [1]. The renewed attention in plastic scintillators has encouraged research towards the modification and improvement of their fundamental properties - namely light yield, decay time, and radiation resistance.

Standard plastic scintillators fluoresce in the blue region of the visible light spectrum and consist of a polymer matrix doped with two fluorescent organic compounds. Polystyrene and poly(vinyltoluene) are common materials utilized as the polymer base. The fluorescent compounds are generally referred to as the primary and secondary dopants. An extensive variety of types and compositions is now commercially available. The new plastic scintillators range in cmissions from the blue to the red region of the visible spectrum. In addition, wavelength shifting fibers, in which only a secondary dopant is present, have been developed to couple to scintillating tiles. Furthermore intrinsic plastic scintillators, where a single fluorescent compound functions as both primary and secondary dopant, are being investigated.

The use of plastic scintillators emitting at longer wavelengths has been dictated by the results from numerous radiation damage tests. These studies indicate that the optical properties of polystyrene degrade with exposure to ionizing radiation $[2,3]$. This effect is observed as an increase of its absorption region towards longer wavelengths and is responsible for losses in the light output of the detector. Since the permanent damage in polystyrene extends up to $500 \mathrm{~nm}$, a dopant fluorescing at wavelengths longer than 500 $\mathrm{nm}$ would yield a plastic scintillator less susceptible to radiation $[4,5]$.

Among the new fluorescent compounds tested, 3-hydroxyflavone (3HF) has been found to be an excellent candidate for green-emitting plastic scintillators. Its absorption and emission peaks are at approximately $350 \mathrm{~nm}$ and $530 \mathrm{~nm}$, respectively. The large separation between absorption and emission spectra is due to an excited-state intramolecular 
proton transfer that occurs when $3 \mathrm{HF}$ is excited by $\mathrm{UV}$ or ionizing radiation. Many $3 \mathrm{HF}$ derivatives have been prepared in an attempt to modify its spectroscopic characteristics to the need of different detectors $[6,7,8]$. Another fluorescent compound which also exhibits excited-state intramolecular proton transfer is 2-(2'-hydroxyphenyl)benzothiazole (HBT). Its absorption and emission wavelengths are similar to those of $3 \mathrm{HF}$. But low quantum yield and questionable radiation resistance render this compound unsuitable for plastic scintillation applications. Nonetheless, HBT remains of interest because $3 H F$ presents problems of oxidation and UV-induced degradation [9] which do not seem to affect the HBT molecule. Different HBT derivatives have been prepared with the aim to solve its intrinsic drawbacks [10].

Here we report the photophysical characteristics of a new set of derivatives that have been prepared at Fermilab. Table 1 presents the list of the compounds studied. Figures 1 and 2 show the schematic of their molecular structures. The substitution has been extended from the phenyl ring to the heteroaromatic moeity where the sulfur atom has been exchanged by either an oxygen or a nitrogen atom, thus preparing $2-\left(2^{\prime}\right.$ hydroxyphenyl)benzoxazole (HBO) or 2-(2'-hydroxyphenyl)benzimidazole (HBI) derivatives, respectively.

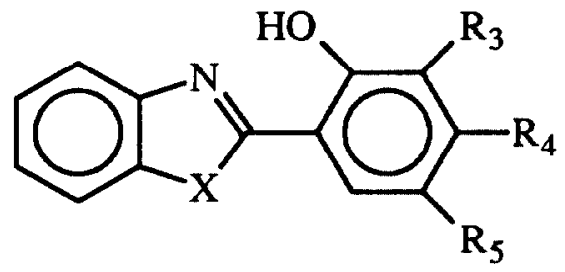

Figure 1. Substitution pattern in $\mathrm{HBT}$, HBO, and HBI.

(B)

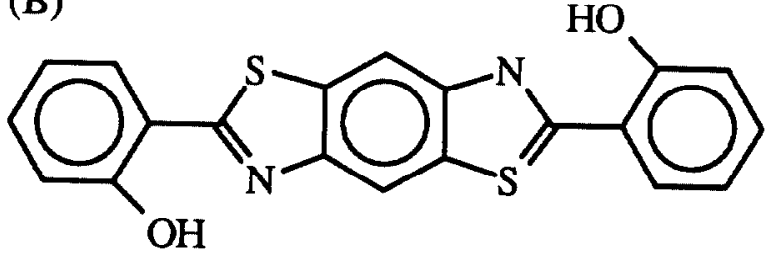

(A)

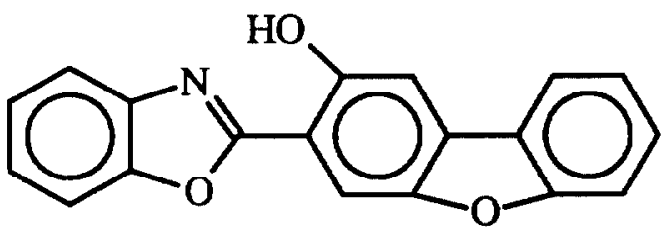

Figure 2. Molecular structures of OX545 (A) and DHBT (B). 
Table 1. List of compounds studied.

\begin{tabular}{cccccl}
\hline \hline $\mathrm{X}$ & $\mathrm{R}_{3}$ & $\mathrm{R}_{\mathbf{4}}$ & $\mathrm{R}_{5}$ & Name & Description \\
\hline $\mathrm{S}$ & $\mathrm{H}$ & $\mathrm{H}$ & $\mathrm{H}$ & $\mathrm{HBT}$ & Benzothiazole \\
$\mathrm{S}$ & $\mathrm{Cl}$ & $\mathrm{H}$ & $\mathrm{H}$ & $3 \mathrm{CHBT}$ & Benzothiazole \\
$\mathrm{S}$ & $\mathrm{H}$ & $\mathrm{H}$ & $\mathrm{Cl}$ & $5 \mathrm{CHBT}$ & Benzothiazole \\
$\mathrm{S}$ & $\mathrm{H}$ & $\mathrm{MeO}$ & $\mathrm{H}$ & $4 \mathrm{MOHBT}^{a}$ & Benzothiazole \\
$\mathrm{O}$ & $\mathrm{H}$ & $\mathrm{H}$ & $\mathrm{H}$ & $\mathrm{HBO}^{a}$ & Benzoxazole \\
$\mathrm{O}$ & $\mathrm{Cl}$ & $\mathrm{H}$ & $\mathrm{H}$ & $3 \mathrm{CHBO}$ & Benzoxazole \\
$\mathrm{O}$ & $\mathrm{H}$ & $\mathrm{H}$ & $\mathrm{Cl}$ & $5 \mathrm{CHBO}$ & Benzoxazole \\
$\mathrm{O}$ & $\mathrm{H}$ & $\mathrm{Ph}$ & $\mathrm{H}$ & $4 \mathrm{PHBO}$ & Benzoxazole \\
$\mathrm{NH}$ & $\mathrm{H}$ & $\mathrm{H}$ & $\mathrm{H}$ & $\mathrm{HBI}$ & Benzimidazole \\
$\mathrm{NH}$ & $\mathrm{Cl}$ & $\mathrm{H}$ & $\mathrm{H}$ & $3 \mathrm{CHBI}$ & Benzimidazole \\
$\mathrm{NH}$ & $\mathrm{H}$ & $\mathrm{H}$ & $\mathrm{Cl}$ & $5 \mathrm{CHBI}$ & Benzimidazole \\
$\mathrm{NH}$ & $\mathrm{H}$ & $\mathrm{Ph}$ & $\mathrm{H}$ & $4 \mathrm{PHBI}$ & Benzimidazole \\
\hline $\mathrm{O}$ & & & & OX545 & Oxazole 545 \\
$\mathrm{S}$ & & & & DHBT & HBT Dimer \\
\hline \hline
\end{tabular}

${ }^{a}$ Commercially available. ${ }^{b}$ Prepared by J.M. Kauffman, Philadelphia College of Pharmacy and Sciences [11].

\section{SAMPLE PREPARATION}

Styrene was deinhibited by passage through a column filled with alumina pellets (F200) available from ALCOA and purified by vacuum distillation. Glass polymerization tubes were cleaned with nitric and sulfuric acids, rinsed with distilled water, dried, and treated for 10 minutes with a $30 \%$ solution of dichlorodimethylsilane in hexane. Finally, they were rinsed in turn with hexane, methanol, and distilled water. This treatment enabled the removal of the plastic once the polymerization cycle was finished. Appropriate dopants were added to the polymerization tubes which were then filled with purified styrene. The various solutions were degassed through repeated freeze-pump-thaw cycles. The solutions were polymerized in a silicone oil bath at $125^{\circ} \mathrm{C}$ for 24 hours and at 140 ${ }^{\circ} \mathrm{C}$ for 48 hours. The bath temperature was ramped down to $90{ }^{\circ} \mathrm{C}$ over 16 hours. After removal from the oil bath, the tubes were quenched in liquid nitrogen for a fast release of the plastic rods. The rods were cut and polished into discs of $2.2 \mathrm{~cm}$ diameter and $1 \mathrm{~cm}$ thick.

\section{FLUORESCENT COMPOUNDS}

The parent compounds, 2-(2'-hydroxyphenyl)benzothiazole (HBT) and 2-(2'-hydroxyphenyl)benzoxazole (HBO), were purchased from Frinton and Aldrich, respectively and further purified by flash chromatography. OX545 was synthesized by J.M. Kauffman (Philadelphia College of Pharmacy and Science) [11] for R.C. Ruchti (University of Notre Dame) and used as received. The remaining derivatives were prepared in our laboratory using different procedures $[12,13,14]$. 
The color of each derivative ranged from white to slightly yellow depending upon the nature and position of the substituent in the phenyl ring. The purity of each derivative was verified using spectroscopic techniques (nuclear magnetic resonance: ${ }^{1} \mathrm{H}$ NMR and ${ }^{13} \mathrm{C}$ NMR), melting point determinations, and Elemental Analysis. The derivatives were used as dopants for intrinsic plastic scintillators in a $1 \%$ by weight concentration to ensure optimum coupling with polystyrene and as wavelength shifters for standard scintillator systems at a $0.01 \%$ by weight concentration. In the latter case, p-terphenyl (PT) was used as the primary dopant at a $1.25 \%$ by weight concentration. Samples containing HBI derivatives were doped only up to $0.2 \%$ by weight because of solubility problems. These samples also used p-terphenyl as the primary dopant. In order to determine the absorptivity coefficients, dilute solutions $\left(10^{-5} \mathrm{M}, \mathrm{M} \equiv \mathrm{mol} /\right.$ Liter $)$ of each derivative in styrene were prepared.

\section{INSTRUMENTATION AND TECHNIQUES}

Transmittance and fluorescence spectra were recorded with a Hewlett-Packard model 8451A diode array spectrophotometer. All absorbance measurements used pure (undoped) polystyrene as the reference. The fluorescence spectra were measured using an external Hg lamp whose light was brought into the spectrophotometer by means of a quartz fiber. Different excitation wavelengths could be selected with the use of bandpass filters. Frontsurface (FS) excitation measurements were performed. Light from the quartz fiber excited the sample surface facing the spectrophotometer collection optics. The sample fluorescence was viewed directly. The angle of incidence with respect to the surface plane was $45^{\circ}$ in this geometry.

Emission time distributions were measured utilizing a ${ }^{22} \mathrm{Na}$ source which excited the

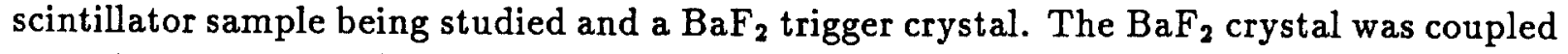
to a photomultiplier (Hamamatsu assembly H3177) and provided the START signal for a LeCroy $q V t$ operating in time mode. The geometry was such that only single photons from the plastic scintillator reached a second photomultiplier (Hamamatsu assembly H4022) which generated the STOP signal. The emission time probability distribution for these scintillators can be described by a one-exponential decay:

$$
E(t)=\frac{1}{\tau_{1}} e^{-t / \tau_{1}}
$$

where $\tau_{1}$ is the decay constant. A gaussian time error with a standard deviation $\sigma_{t}$ was assumed. The resulting time distribution is then:

$$
P(t)=\int_{0}^{\infty} E\left(t^{\prime}\right) \frac{1}{\sqrt{2 \pi} \sigma_{t}} e^{-\left(t-t^{\prime}\right) / 2 \sigma_{t}^{2}} d t^{\prime}
$$

By fitting the above distribution to the data, the $\tau_{1}$ parameter is determined.

The samples for radiation damage studies were placed in stainless steel cans and then evacuated for two weeks. The cans were then back-filled with dry nitrogen. All samples were irradiated with a ${ }^{60}$ Co source at the Phoenix Memorial Laboratory of the University 
of Michigan at a rate of approximately $1.7 \mathrm{Mrad} / \mathrm{h}$ to a total dose of $10 \mathrm{Mrad}$. All the samples developed some coloration during the exposure, some of which disappeared with time when the samples were exposed to oxygen. For this study, the samples were kept under a 100 psig. oxygen atmosphere for 8 days after their irradiation.

\section{RESULTS AND DISCUSSION}

All the HBT, HBO, and HBI derivatives studied exhibit excited-state intramolecular proton transfer (ESIPT) upon excitation by UV or ionizing radiation. Figure 3 illustrates this mechanism in the HBT molecule. The proton transfer causes a structural rearrangement, which is indicated by the large separation between the absorption and the fluorescent bands. The proton transfer is favored because of an increase in the $\mathrm{N}$ basicity and the $O$ acidity in the excited state and, in addition, it is aided by a pre-existing hydrogen bond.

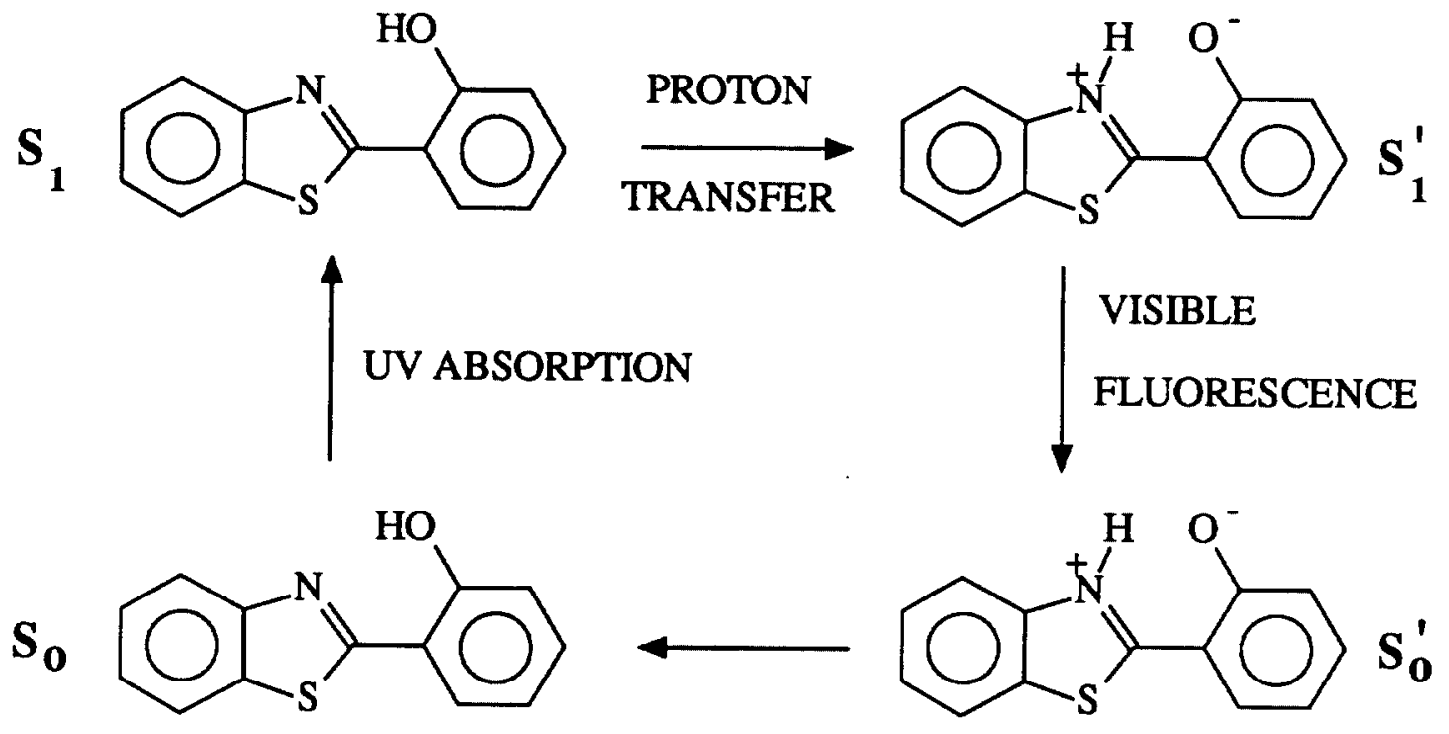

Figure 3. Excited-state intramolecular proton transfer process for HBT.

The first compounds prepared were the HBT derivatives. Radiation damage studies performed on scintillator samples doped with these derivatives indicated the formation of a new and permanent absorption band at longer wavelengths. Figure 4 presents these results for HBT. This second absorption band has been assigned to the formation during irradiation of the keto isomer [10,14]. The presence of this isomer reduces the effective separation between absorption and emission spectra after irradiation and causes light yield losses due to self-absorption. The $\mathrm{HBO}$ and $\mathrm{HBI}$ derivatives were synthesized in an attempt to find a compound that would still exhibit ESIPT but would not form isomers under radiation. Figure 5 shows the transmittance and fluorescence spectra for the parent compounds (HBT, HBO, and HBI). 

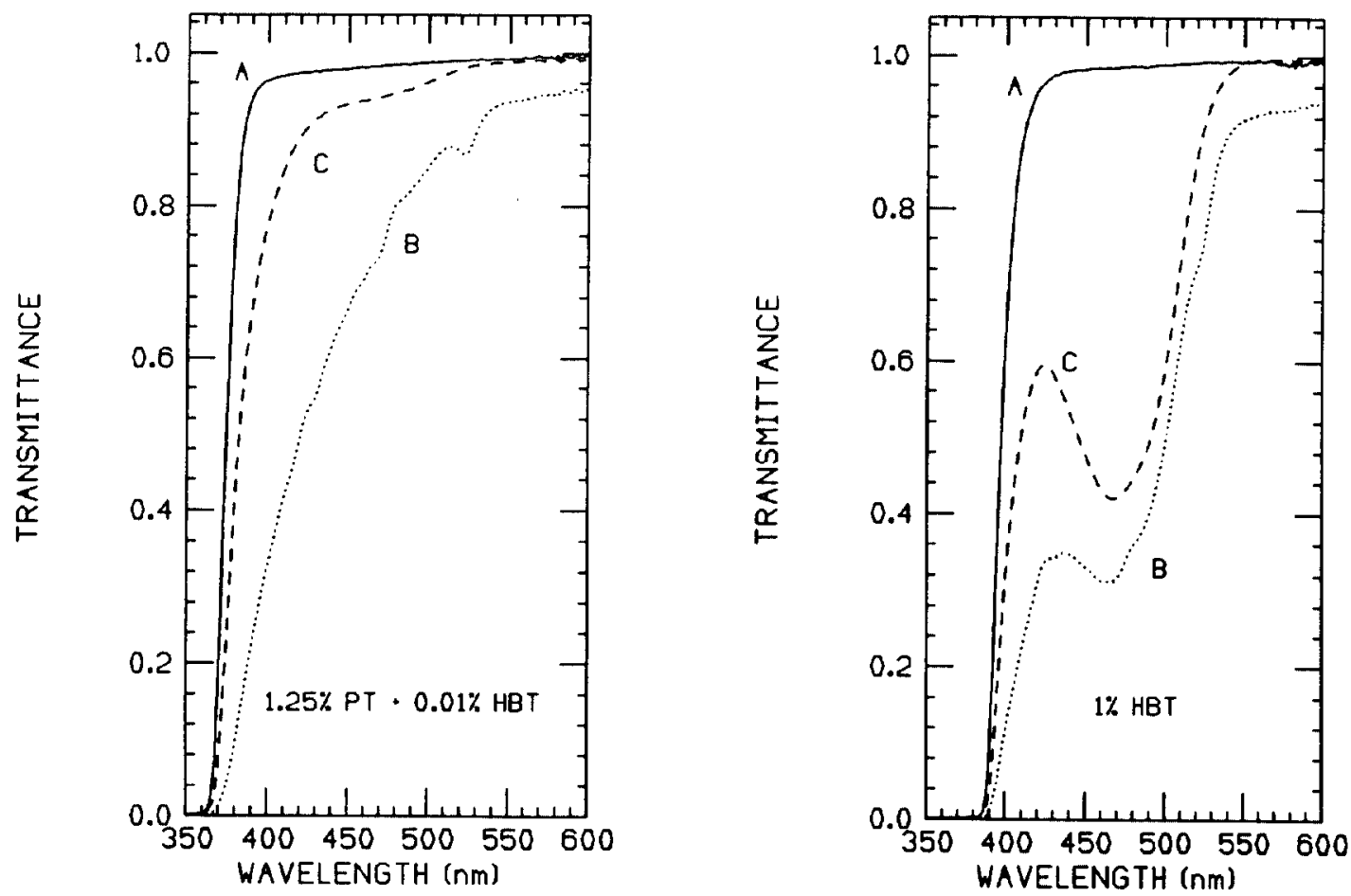

Figure 4. Irradiation of HBT-doped polystyrene samples: (A) before irradiation, (B) after $10 \mathrm{Mrad}$, and (C) after oxygen annealing.
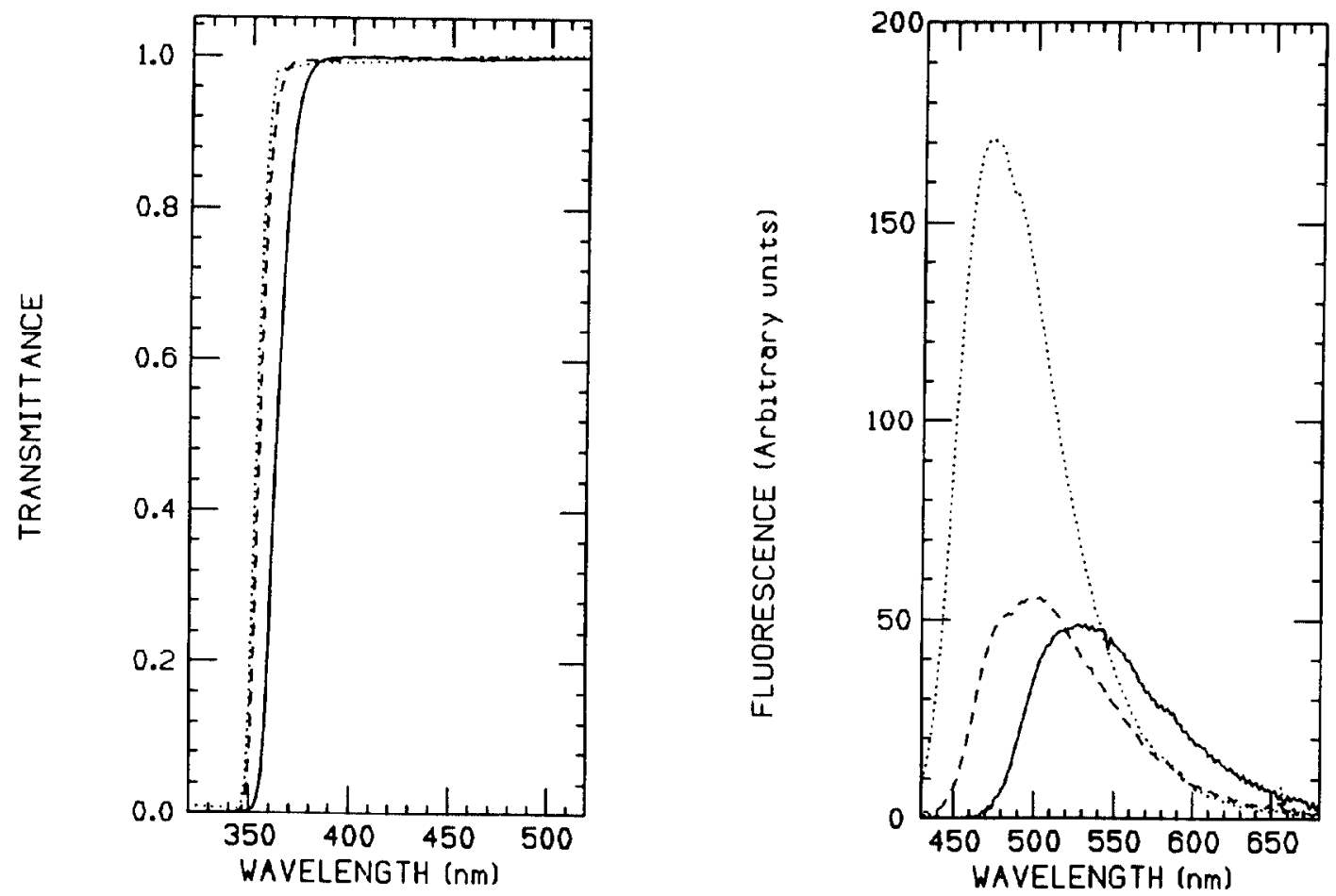

Figure 5. Transmittance and fluorescence spectra of $1.25 \% \mathrm{PT}+0.01 \% \mathrm{HBT}$ (solid), $1.25 \% \mathrm{PT}+0.01 \% \mathrm{HBO}$ (dashes), and $1.25 \% \mathrm{PT}+0.01 \% \mathrm{HBI}$ (dots). 
Table 2 lists the photophysical characteristics of the compounds studied including representative $\mathrm{HBT}$ derivatives, and $3 \mathrm{HF}$ for a base reference. The absorption and emission curves of the HBO and HBI derivatives show a hypsochromic shift with respect to those of the HBT derivatives. In the absorption spectra, the shift is approximately $10 \mathrm{~nm}$ towards shorter wavelengths; and in the fluorescence spectra, it is about $30 \mathrm{~nm}$ for HBO derivatives and $60 \mathrm{~nm}$ for HBI derivatives. These spectral differences were anticipated, although the Stokes-shift reduction of the HBI derivatives was not expected to be as significant. However, the HBI derivatives now offer the possibility to act as primary dopants with a large Stokes shift. A primary dopant with these characteristics may be useful even though there are already many secondary dopants fluorescing in the blue/green region.

Table 2. Photophysical characteristics of the derivatives in polystyrene.

\begin{tabular}{lccccc}
\hline \hline Compound $^{a}$ & $\begin{array}{c}\lambda_{a b s}^{b} \\
(\mathrm{~nm})\end{array}$ & $\begin{array}{c}\varepsilon^{c} \\
\left(\mathrm{~L} \mathrm{~mol}^{-1} \mathrm{~cm}^{-1}\right)\end{array}$ & $\begin{array}{c}\lambda_{e m}^{d} \\
(\mathrm{~nm})\end{array}$ & $\begin{array}{c}\text { Relative } \\
\text { Quantum Yield }\end{array}$ & $\begin{array}{c}\text { Decay time } \\
\tau \text { (ns) }\end{array}$ \\
\hline $0.01 \%$ 3HF & 350 & 15000 & 530 & 1.0 & $8.5 \pm 0.1$ \\
$0.01 \%$ HBT & 340 & 15000 & 530 & 0.4 & $3.9 \pm 0.1$ \\
$0.01 \%$ 3CHBT & 344 & 16000 & 540 & 0.6 & $4.8 \pm 0.1$ \\
$0.01 \%$ 5CHBT & 350 & 17000 & 542 & 0.6 & $4.8 \pm 0.1$ \\
$0.01 \%$ 4MOHBT & 342 & 23000 & 550 & 0.4 & $3.7 \pm 0.1$ \\
$0.01 \%$ HBO & 330 & 16000 & 498 & 0.4 & $3.6 \pm 0.1$ \\
$0.01 \%$ 3CHBO & 332 & 14000 & 510 & 0.6 & $4.2 \pm 0.1$ \\
$0.01 \%$ 5CHBO & 340 & 15000 & 510 & 0.7 & $5.1 \pm 0.1$ \\
$0.01 \%$ 4PHBO & 340 & 30000 & 510 & 0.8 & $4.8 \pm 0.1$ \\
$0.20 \%$ HBI & 330 & 18000 & 470 & 1.1 & $5.2 \pm 0.1$ \\
$0.20 \%$ 3CHBI & 332 & 20000 & 480 & 1.1 & $5.6 \pm 0.1$ \\
$0.20 \%$ 5CHBI & 340 & 21000 & 490 & 1.2 & $6.2 \pm 0.1$ \\
$0.20 \%$ 4PHBI & 340 & 26000 & 490 & 1.1 & $4.7 \pm 0.1$ \\
$0.01 \%$ OX545 & 336,370 & 32000,20000 & 540 & 1.1 & $8.7 \pm 0.1$ \\
$0.01 \%$ DHBT & 370,398 & 38000,34000 & 542 & 0.7 & $4.5 \pm 0.1$ \\
\hline \hline
\end{tabular}

${ }^{a}$ Samples contain $1.25 \%$ PT as primary dopant. ${ }^{b}$ Maximum absorption wavelength (from diluted styrene solutions). ${ }^{C}$ Absorptivity coefficient (from diluted styrene solutions). ${ }^{d}$ Maximum emission wavelength. ${ }^{e} \mathrm{Quantum}$ yield values relative to $3 \mathrm{HF}$ (from measurements using 334-nm excitation wavelength). ${ }^{f}$ LP 400 filter used to remove non-absorbed light from PT.

Fluorescence measurements were performed using a $334-\mathrm{nm}$ excitation wavelength. The emission spectrum was integrated over all wavelengths to estimate the relative quantum yield of each compound. Light yields have been calculated relative to $3 \mathrm{HF}$ since it is a widely used green dopant. The light yield of the HBO derivatives is similar to that of the HBT derivatives and too low for practical considerations. The light yield of the HBI derivatives is significantly higher. The lifetimes were determined between 4-6 ns. This range is within the desirable limits, for the current goal is to develop dopants faster than the existing green ones (decay times between 7-12 ns). 
OX545 and DHBT are more complex benzoxazole and benzothiazole derivatives. Their preparation involves a multi-step reaction process. OX545 shows that given the proper substituents, the fluorescence spectra of the HBO derivatives can be shifted towards longer wavelengths, to the region of interest. In addition, its light output and decay time have increased to the level of $3 \mathrm{HF}$. The DHBT derivative can be considered a dimeric form of HBT. Therefore it has two sites that can undergo ESIPT. If there is double proton transfer, the fluorescence spectrum will be at longer wavelengths. From the spectroscopic data gathered, DHBT seems to show only single proton transfer. Its fluorescence distribution is similar to that of HBT. The main difference from its parent compound is that its quantum yield is significantly higher. The lifetime of the DHBT derivative remains under $5 \mathrm{~ns}$.

Radiation damage studies have been carried out for the chloro-substituted $\mathrm{HBO}$ and HBI derivatives, OX545, and DHBT. The samples show a large increase in absorption immediately after irradiation. This absorption is largely transient and disappears with time. This process is accelerated by annealing the samples in an oxygen atmosphere. As observed in other scintillators, the samples present certain permanent damage since a residual absorption remains. However, there are no clear signs of the formation of a new band. Figures 6 and 7 present the transmittance data for HBO and HBI. A more detailed analysis should be performed for DHBT, since only low concentration samples were irradiated for this study.
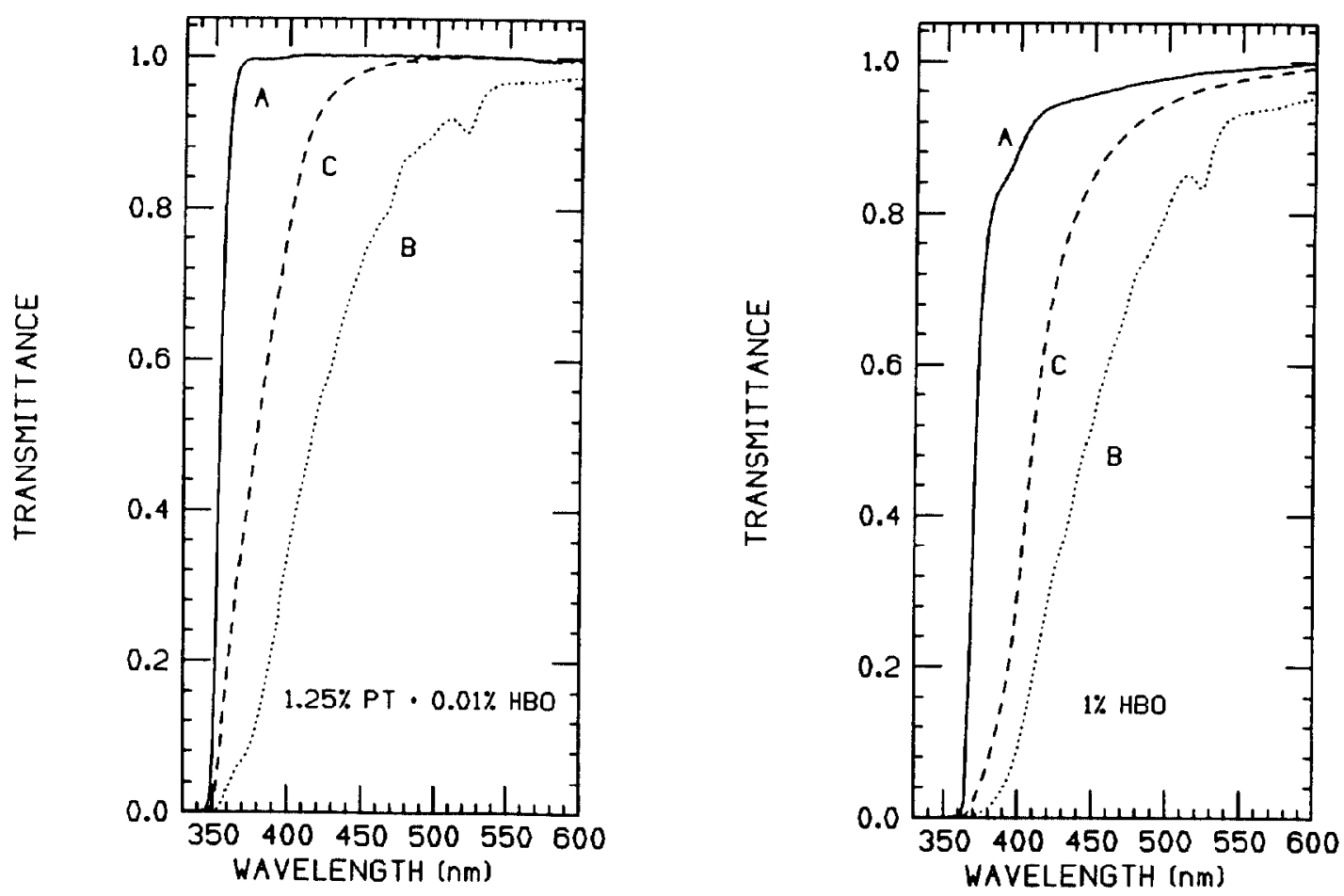

Figure 6. Irradiation of HBO-doped polystyrene samples: (A) before irradiation, (B) after $10 \mathrm{Mrad}$, and (C) after oxygen annealing. 

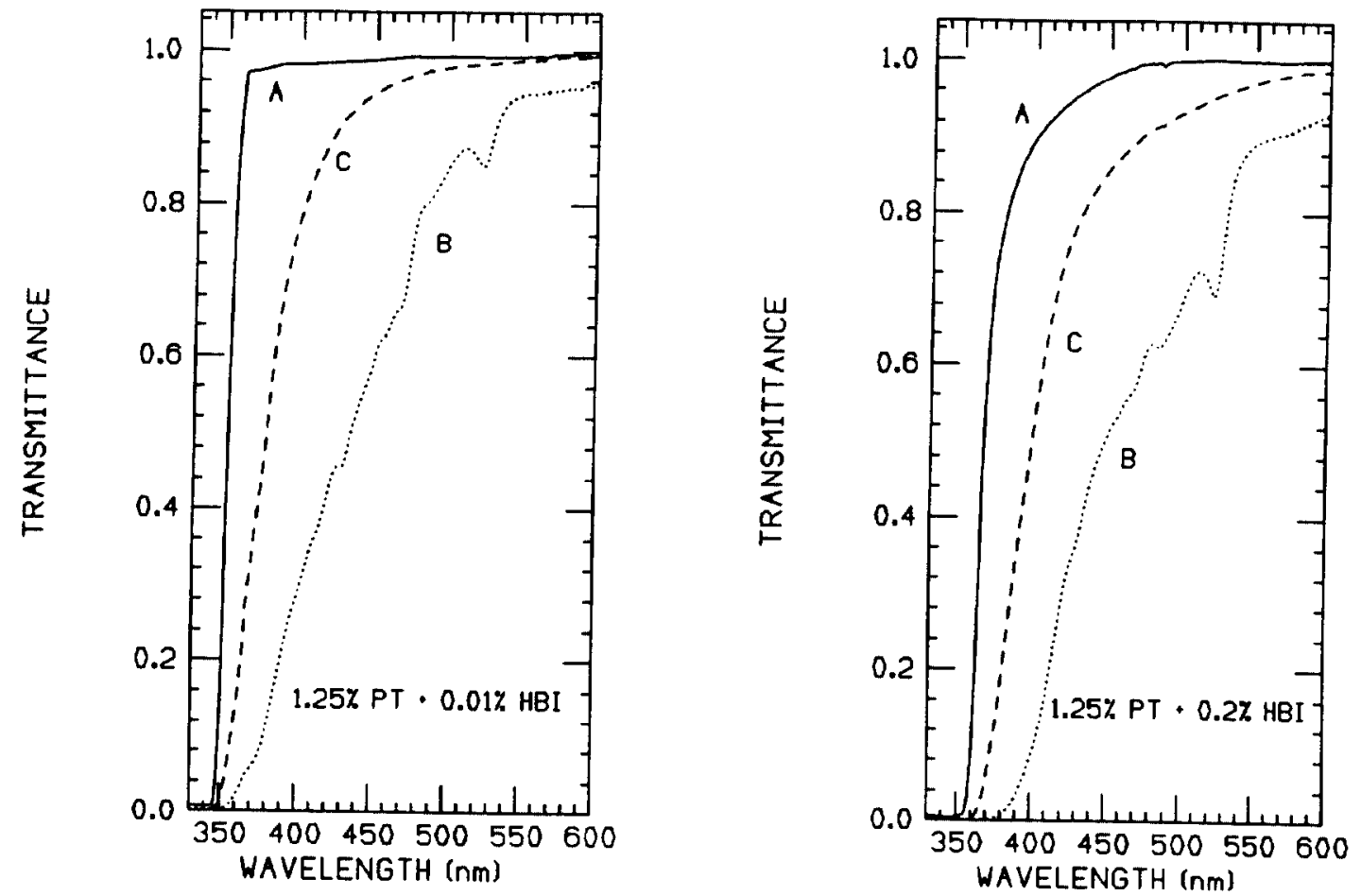

Figure 7. Irradiation of HBI-doped polystyrene samples: (A) before irradiation, (B) after 10 Mrad, and (C) after oxygen annealing.

\section{CONCLUSIONS}

The HBT derivatives have short lifetimes, low quantum yield, and poor radiation resistance due to isomer formation under such conditions. The DHBT derivative presents an improvement over these compounds but further work is required to completely determined its behaviour under radiation. The HBO derivatives present similar characteristics with the important exception of the radiation-induced isomer formation. Nonetheless, OX545 proves that it is possible to increase the quantum yield and shift the fluorescence to the green region of the visible spectrum. The HBI derivatives show a good combination of decay time, light yield, and radiation resistance. However, because of the their emission range they are better candidates for primary rather than for secondary dopants. In order to test them as primary dopants, their solubility in styrene needs to be improved. New HBI derivatives are being prepared to solve this problem.

\section{ACKNOWLEDGEMENTS}

We thank Professors R.C. Ruchti (University of Notre Dame) and J.M. Kauffman (Philadelphia College of Pharmacy and Science) for their gift of Oxazole 545 (OX545). We gratefully acknowledge R. Blackburn (University of Michigan) for performing all sample irradiations. This work was supported by the U.S. Department of Energy under contract No. DE-AC02-76CHO3000. 


\section{REFERENCES}

1. Proceedings of the Workshop on Scintillating Fiber Detectors, edited by R.C. Ruchti, M. Wayne, and A.D. Bross (World Scientific, Singapore, to be published).

2. J.S. Wallace, M.B. Sinclair, K.T. Gillen, and R.L. Clough, Radiat. Phys. Chem. 41 $(1 / 2), 85(1993)$.

3. A.D. Bross and A. Pla-Dalmau in Radiation Effects on Polymers, edited by R.L. Clough and S.W. Shalaby (ACS Symposium Series No. 475, 1991) pp. 578-590.

4. C. Zorn, IEEE Trans. Nucl. Sc. 36, 579 (1989).

5. K.F. Johnson et al., IEEE Trans. Nucl. Sc. 37, 500 (1990).

6. J.R. Dharia, K.F. Johnson, and J.B. Schlenoff, Polym. Preprints 33 (2), 186 (1992); Macromolecules, to be published.

7. J.M. Kauffman and M.A. Aziz, J. Heterocyclic Chem. 30, 1549 (1993).

8. A.D. Bross, A. Pla-Dalmau, and C.W. Spangler, Nucl. Instr. Meth. A235, 168 (1993).

9. M. Chung and S. Margulies in Scintillating Fiber Technology and Applications, edited by E.J. Fenyves (SPIE Proc. 41, 2007 (1993)).

10. A.D. Bross, A. Pla-Dalmau, and C.W. Spangler, Radiat. Phys. Chem. 41 (1/2), 379 (1993).

11. J.M. Kauffman, Proceedings of the Workshop on Scintillating Fiber Detectors, edited by R.C. Ruchti, M. Wayne, and A.D. Bross (World Scientific, Singapore, to be published).

12. K. Anthony et al., J. Chem. Soc. Perkin Trans. $2 \underline{1984}, 2111$.

13. D.W. Hein et al., U.S. Patent No. 2985661 (23 May 1961).

14. J.F. Wolfe, B.H. Loo, and F.E. Arnold, Macromolecules 14, 915 (1981).

15. W.E. Brewer, M.L. Martinez, and P-T. Chou, J. Phys. Chem. 84, 1915 (1990). 